\title{
Growth, strain, and spin-orbit torques in epitaxial Ni-Mn-Sb films sputtered on GaAs
}

\author{
N. Zhao, ${ }^{1}$ A. Sud,${ }^{1}$ H. Sukegawa $\odot,{ }^{2}$ S. Komori, ${ }^{3}$ K. Rogdakis $\odot,{ }^{1}$ K. Yamanoi $\odot,{ }^{1}$ J. Patchett,,${ }^{4}$ J. W. A. Robinson, ${ }^{3}$ \\ C. Ciccarelli, ${ }^{4, *}$ and H. Kurebayashi ${ }^{1, \dagger}$ \\ ${ }^{1}$ London Centre for Nanotechnology, University College London, 17-19 Gordon Street, London WC1H 0AH, United Kingdom \\ ${ }^{2}$ Research Center for Magnetic and Spintronic Materials, National Institute for Materials Science, 1-2-1 Sengen, Tsukuba 305-0047, Japan \\ ${ }^{3}$ Department of Materials Science and Metallurgy, University of Cambridge, 27 Charles Babbage Road, \\ Cambridge CB3 OFS, United Kingdom \\ ${ }^{4}$ Cavendish Laboratory, University of Cambridge, Cambridge CB3 OHE, United Kingdom
}

(Received 15 September 2020; revised 3 December 2020; accepted 8 January 2021; published 25 January 2021)

\begin{abstract}
We report current-induced spin torques in epitaxial NiMnSb films on a commercially available epiready GaAs substrate. The $\mathrm{NiMnSb}$ was grown by cosputtering from three targets using optimized parameters. The films were processed into microscale bars to perform current-induced spin-torque measurements. Magnetic dynamics were excited by microwave currents, and electric voltages along the bars were measured to analyze the symmetry of the current-induced torques. We found that the extracted symmetry of the spin torques matches those expected from spin-orbit interaction in a tetragonally distorted half-Heusler crystal. Both fieldlike and dampinglike torques are observed in all the samples characterized, and the efficiency of the current-induced torques is comparable to that of ferromagnetic metal/heavy-metal bilayers.
\end{abstract}

DOI: 10.1103/PhysRevMaterials.5.014413

\section{INTRODUCTION}

In crystals with broken inversion symmetry, a nonequilibrium spin polarization can be generated by an electric current. This is termed the Edelstein effect [1,2] (or inverse spingalvanic effect [3]), which was discovered in III-V compound semiconductors [4] that lack an inversion center in their zincblende crystal unit cell. This current-induced nonequilibrium spin polarization can be used to manipulate magnetic moments in noncentrosymmetric magnetic crystals [5-12] and also in low-symmetry interfaces $[13,14]$. Akin to the Edelstein effect where an electric current generates a uniform spin polarization across the entire sample, the spin-Hall effect (SHE) [15-20] can generate spin currents from electric charge currents. This mechanism can be exploited to exert magnetic torques within multilayers of ferromagnetic and heavy high spin-orbit coupling metals [21,22]. These two types of spin torques originate from the spin-orbit transport effects, often termed spin-orbit torques, and have been widely studied in many different film stacks [23-37].

Although metallic multilayers are more common in the study of spin-orbit torques, noncentrosymmetric magnetic crystals do play a pivotal role in advancing the research field. Since spin-orbit torques in noncentrosymmetric magnetic crystals are generated within a single layer of a material and not at an interface, effects due to interfaces of the sample such as the SHE can be ruled out. In practice this makes the analysis of the experimental results simpler. For example, a sizable dampinglike component of the spin-orbit torques in

\footnotetext{
*cc538@cam.ac.uk

†h.kurebayashi@ucl.ac.uk
}

GaMnAs was discovered [10], which might have been difficult to identify in metallic multilayers because of the SHE that gives a strong dampinglike component in multilayers [21,22]. In addition, the direction of the current-induced spin polarization in noncentrosymmetric magnetic crystals is determined by the point group of the crystal [11,38,39], which leads to a variety of torque symmetries that depend on the choice of material.

In this paper, we characterize spin-orbit torques in sputterdeposited epitaxial $\mathrm{NiMnSb}$ thin films. $\mathrm{NiMnSb}$ is a room temperature ferromagnet [40] and half-Heusler compound that has been studied for its predicted half metallicity [41] as well as high magnetic tunability via stoichiometry [42]. Ciccarelli et al. [11] discovered spin-orbit torques in epitaxial $\mathrm{NiMnSb}$ on lattice-matched InGaAs, which was tailor-made in a III-V molecular beam epitaxy (MBE) chamber. In our study, we prepared epitaxial NiMnSb thin films by sputtering on commercial epitaxial GaAs. Our structural analysis reveals a tetragonal distortion in the epitaxial $\mathrm{NiMnSb}$ films due to the growth-induced strain from the GaAs substrates. By using current-induced ferromagnetic resonance (FMR) experiments, we identified spin-orbit torques arising from the strain-induced spin-orbit Hamiltonian. The NiMnSb thin films exhibit torques five times larger than those measured in NiMnSb grown by MBE [11], matching the performance of ferromagnetic metal/heavy-metal bilayers. Furthermore, epitaxial sputtered NiMnSb shows strong dampinglike spin-orbit torques, which are absent in MBE NiMnSb [11].

\section{SAMPLE GROWTH AND FILM CHARACTERIZATION}

A NiMnSb film was deposited on a $\mathrm{GaAs}(001)$ substrate by a magnetron cosputtering system with a base pressure of 
(a)
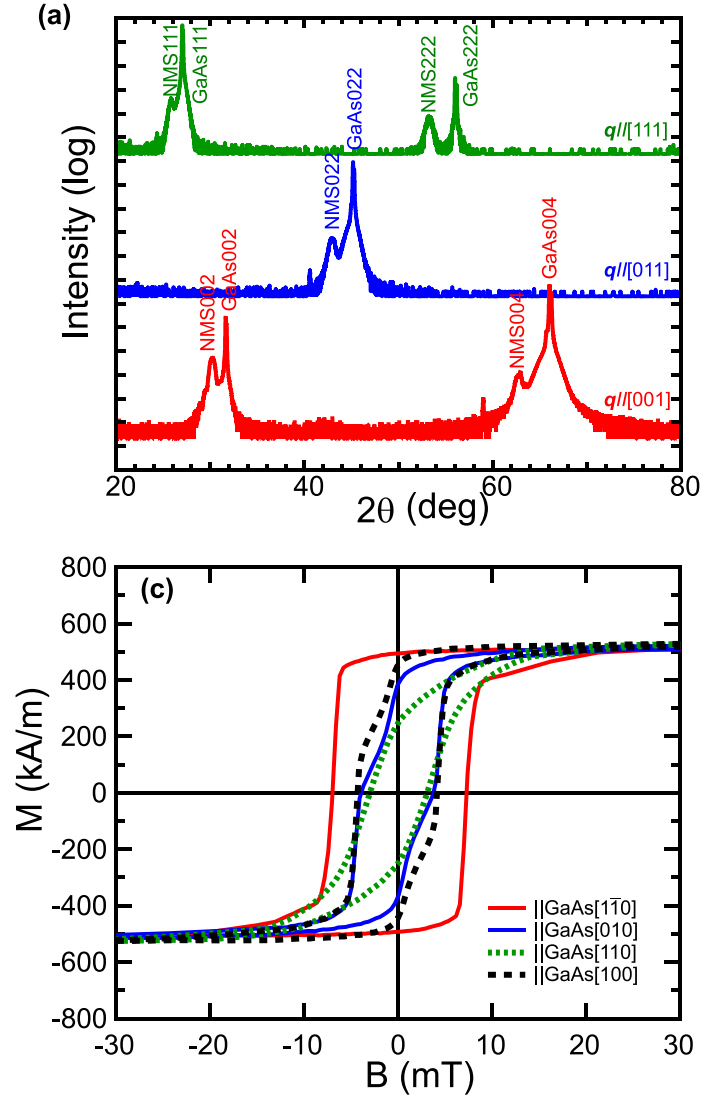
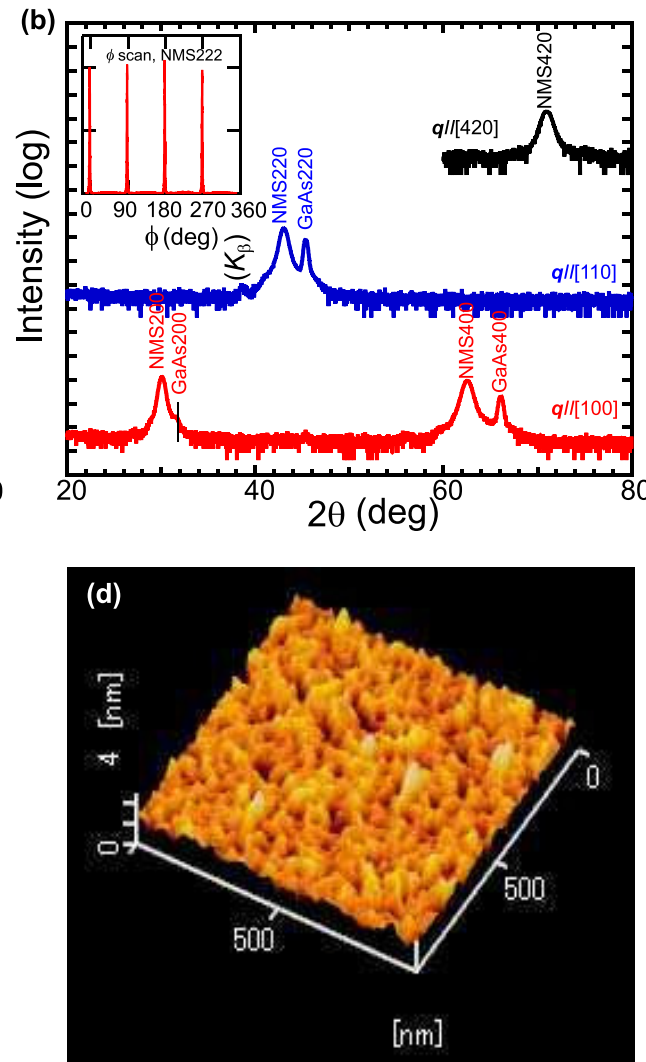

FIG. 1. (a) and (b) XRD profiles for a GaAs(001) substrate/NiMnSb (NMS) $17 \mathrm{~nm} / \mathrm{MgO} 3 \mathrm{~nm}$ film. (a) Out-of-plane and (b) in-plane profiles along various scattering vectors $q$ of GaAs. The inset of (b) shows the NMS222 $\phi$ scan profile (pole figure). (c) In-plane $M$ - $H$ curves along GaAs[110], [010], [110], and [100]. (d) An AFM image of the $17 \mathrm{~nm}$ thick NiMnSb film used in the present study.

$1 \times 10^{-7} \mathrm{~Pa}$. Prior to each deposition, the GaAs substrate was cleaned with a $35 \%$ hydrochloric acid $(\mathrm{HCl})$ aqueous solution at room temperature for $9 \mathrm{~min}$ before being transferred into the sputtering chamber. Surface cleaning by annealing at $400{ }^{\circ} \mathrm{C}$ for $30 \mathrm{~min}$ was performed, followed by a sample cooling process down to the growth temperature of $250^{\circ} \mathrm{C}$. The $\mathrm{NiMnSb}$ films were grown by cosputtering from $\mathrm{Ni}, \mathrm{Mn}$, and $\mathrm{Sb}$ targets where the sputtering powers for individual targets were tuned to control the sample stoichiometry close to the desired 1:1:1 ratio of each element. The NiMnSb deposition rate was set to $0.12 \mathrm{~nm} / \mathrm{s}$, and the deposition was performed using an Ar partial pressure of 0.4 $\mathrm{Pa}$. $\mathrm{Mn}$ and $\mathrm{Sb}$ were deposited by dc sputtering, whereas Ni was deposited by rf sputtering. We grew samples with different NiMnSb thicknesses for growth optimization, and for this particular study, we used a 17nm-thick NiMnSb film to fabricate micron-scale bar devices. After the deposition, the films were postannealed at $400{ }^{\circ} \mathrm{C}$ within the chamber. Finally, a 3-nm-thick $\mathrm{MgO}$ layer was deposited by rf sputtering at room temperature. We confirm that the $\mathrm{MgO}$ layer acts as a capping layer by resistivity measurements of NiMnSb films. The composition of $\mathrm{NiMnSb}$ was measured as $\mathrm{Ni}_{31} \mathrm{Mn}_{33} \mathrm{Sb}_{36}$ by inductively coupled plasma analysis of a 100-nm-thick reference film deposited on a thermally oxidized $\mathrm{Si}$ substrate at room temperature. The crystal structure was characterized by four-axis X-ray diffraction (XRD; Rigaku Smartlab) with $\mathrm{Cu} K \alpha$ radiation and a graphite diffracted beam monochromator. The magnetic hys- teresis loops $(M-H$ loops) were measured using a vibrating sample magnetometer (VSM) at room temperature. The surface morphology was evaluated by atomic force microscopy (AFM). Figure 1 shows a summary of our film characterization results. Figures 1(a) and 1(b) trace the XRD profiles for out-of-plane $(2 \theta-\omega$ scans $)$ and in-plane $\left(2 \theta_{\chi}-\phi\right.$ scans $)$ rotations, respectively, with different incident directions. The inset of Fig. 1(b) shows the $\phi$ scan for a NiMnSb(222) plane. These traces confirm that the NiMnSb film is epitaxially grown on GaAs with cube-on-cube orientation relationship of $\mathrm{GaAs}(001) / / \mathrm{NiMnSb}(001)$ and GaAs[100]//NiMnSb[100], which is consistent with previously reported $\mathrm{NiMnSb}$ films on GaAs [43]. No secondary phases were detected in the profiles, indicating formation of a single $C 1_{b}$-type $\mathrm{NiMnSb}$ phase. The $00 l$ and $0 l l$ peak angles (out-of-plane) are slightly lower than the $l 00$ and $l l 0$ angles (in-plane), respectively. Therefore, the NiMnSb film is tetragonally distorted. Using the peaks' positions and the least-squares method, we obtained lattice constants with standard deviations $a=b=$ $0.5937 \pm 0.0002 \mathrm{~nm}$ and $c=0.5921 \pm 0.0002 \mathrm{~nm}$, giving $c / a=0.997 \pm 0.0002$. The sign of this distortion generated by growth-induced strain is opposite to the lattice mismatch of their bulk lattice constants: the bulk value of the lattice constants is $0.593 \mathrm{~nm}$ for NiMnSb [44] and $0.5653 \mathrm{~nm}$ for GaAs [45], for which we would expect the NiMnSb crystal grown on $\mathrm{GaAs}$ to have a compressive strain of about $4.9 \%$. We speculate that misfit dislocations are periodically introduced 
near the GaAs/NiMnSb interface, which greatly influences the lattice relaxation. The residual tetragonal distortion that we measured in the $\mathrm{x}$-ray scans can be determined by both residual sputter-deposition stress and lattice mismatches. Figure 1 (c) displays the in-plane $M-H$ curves along [1피, [010], [110], and [100] in GaAs. The shape of the different crystallographic directions suggests the presence of both uniaxial and cubic magnetic anisotropies (we will discuss more detailed results for these below). The extracted saturation magnetization is $668 \pm 17 \mathrm{mT}$, which is about 0.7 times smaller than the bulk value $(930 \mathrm{mT})$ [46]. The saturation magnetization of $\mathrm{NiMnSb}$ is sensitive to growth conditions $[47,48]$ and the stoichiometry of NiMnSb. A similar reduction in epitaxial NiMnSb thin films was reported by Kwon et al. [49]. Possible causes for the reduction include (i) a smaller concentration of $\mathrm{Ni}$ and $\mathrm{Mn}$ atoms with respect to $\mathrm{Sb}$; (ii) the existence of a magnetically dead layer at the GaAs/NiMnSb and $\mathrm{NiMnSb} / \mathrm{MgO}$ interfaces, and (iii) residual antisite defects in the $C 1_{b}$ lattice. As shown in Fig. 1(d), the AFM micrograph $\left(1 \times 1 \mu \mathrm{m}^{2}\right.$ area $)$ of the NiMnSb surface reveals the mean average roughness of $R a=0.31 \mathrm{~nm}$ and the maximum peak-to-valley value of $4.8 \mathrm{~nm}$ from this characterization. A key challenge of our growth optimization was to maintain the high crystallinity of NiMnSb films, with minimal roughness. The crystallinity was improved by increasing the growth temperature up to $400^{\circ} \mathrm{C}$, at the expense of roughness. Therefore, we grew the NiMnSb at $250{ }^{\circ} \mathrm{C}$ with postannealing at $400^{\circ} \mathrm{C}$.

\section{DEVICE FABRICATION AND MEASUREMENT TECHNIQUES}

Each $\mathrm{NiMnSb}$ film was cut into 5-mm square chips for device fabrication by optical lithography into bars. A device schematic together with the electric circuitry of the measurement setup is shown in Fig. 2(a). The NiMnSb bar width and length are 5 and $50 \mu \mathrm{m}$, respectively. We use the phenomenological Landau-Lifshitz-Gilbert equation to describe the spin-orbit-induced magnetization dynamics in our experiments:

$$
\frac{\partial \boldsymbol{M}}{\partial t}=-\gamma \boldsymbol{M} \times \boldsymbol{H}_{\mathrm{tot}}+\frac{\alpha}{M_{\mathrm{s}}}\left(\boldsymbol{M} \times \frac{\partial \boldsymbol{M}}{\partial t}\right)-\gamma \boldsymbol{M} \times \boldsymbol{h}_{\mathrm{so}} .
$$

Here, $\gamma$ is the gyromagnetic ratio, and $\alpha$ is the phenomenological Gilbert damping constant. $\boldsymbol{H}_{\text {tot }}$ is the total magnetic field vector, and $\boldsymbol{h}_{\text {so }}$ is the effective spin-orbit magnetic field that drives the magnetic moments. Within a small precession angle approximation, where the magnetization dynamics is in the linear excitation regime, we can write $\boldsymbol{M}=\left(M_{\mathrm{s}}, m_{b} e^{i \omega t}, m_{c} e^{i \omega t}\right)$, where $M_{\mathrm{s}}$ represents the saturation magnetization. We focus on the alternating in-plane angle $\left[m_{b}(t) / M_{\mathrm{s}}\right]$ since this motion leads to rectified voltages [10]. This magnetization precession causes a time-varying resistance change originating in the anisotropic magnetoresistance: $R(\theta)=R_{0}-\Delta R \cos ^{2}(\theta)$, where $R_{0}$ and $\Delta R$ are magnetization-angle-independent and -dependent terms, respectively, and $\theta$ is defined by the angle between the current flowing direction and static magnetization orientation. This time-varying resistance change is mixed with a time-varying electric current component (which exerts spin torques in our $\mathrm{NiMnSb}$ films), providing experimentally measurable recti- (a)

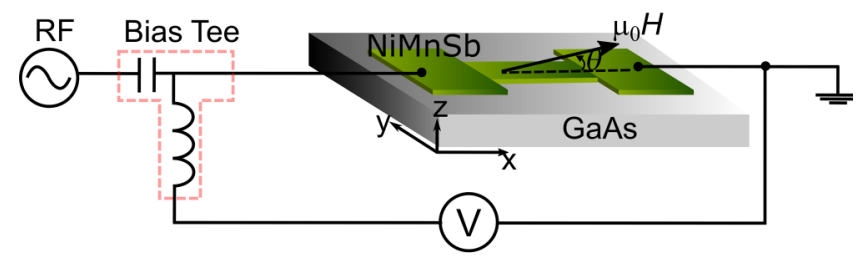

(b)

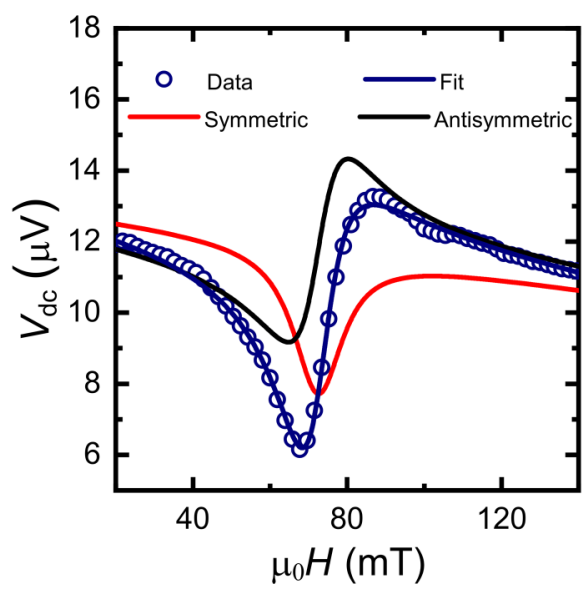

FIG. 2. (a) Schematic of the measurement setup using this study with circuit configuration. (b) Typical FMR voltages measured at a microwave excitation frequency of $7 \mathrm{GHz}$ with the external magnetic field applied along $\theta=60^{\circ}$ in the [110] device. Dots are experimental data points, and curves are produced by best-fit parameters using Eq. (2) and voltage offsets that are constant with field and linear to field.

fication voltages in the form of $V_{\mathrm{dc}}=\left(I \Delta R m_{b} / 2 M_{\mathrm{s}}\right) \sin 2 \theta$ [10]. In this model, the real component of $V_{\mathrm{dc}}$ is decomposed in the sum of symmetric and asymmetric Lorentzians [9]:

$$
\begin{aligned}
\operatorname{Re}\left\{V_{\mathrm{dc}}\right\}= & V_{\text {sym }} \frac{\Delta H^{2}}{\left(H-H_{\text {res }}\right)^{2}+\Delta H^{2}} \\
& +V_{\text {asy }} \frac{\Delta H\left(H-H_{\text {res }}\right)}{\left(H-H_{\text {res }}\right)^{2}+\Delta H^{2}} .
\end{aligned}
$$

Here, $H_{\text {res }}, H$, and $\Delta H$ are the resonance field for FMR, applied magnetic field, and the linewidth of FMR peaks, respectively.

$$
\begin{aligned}
V_{\text {sym }}(\theta)= & \frac{I \Delta R \omega}{2 \gamma \Delta H\left(2 H_{\text {res }}+H_{1}+H_{2}\right)} \sin (2 \theta) h_{z}, \\
V_{\text {asy }}(\theta)= & \frac{I \Delta R\left(H_{\text {res }}+H_{1}\right)}{2 \Delta H\left(2 H_{\text {res }}+H_{1}+H_{2}\right)} \\
& \times \sin (2 \theta)\left(-h_{x} \sin \theta+h_{y} \cos \theta\right)
\end{aligned}
$$

are the weights that determine the line shape of the resonance which will be used for quantifying the components of the spinorbit effective field $h_{x}, h_{y}$, and $h_{z} . H_{1}$ and $H_{2}$ are

$$
\begin{gathered}
H_{1}=M_{s}-H_{2 \perp}+H_{2 \|} \cos ^{2}\left(\phi+\frac{\pi}{4}\right) \\
+\frac{1}{4} H_{4 \|}(3+\cos 4 \phi), \\
H_{2}=H_{4 \|} \cos 4 \phi-H_{2 \|} \sin 2 \phi .
\end{gathered}
$$




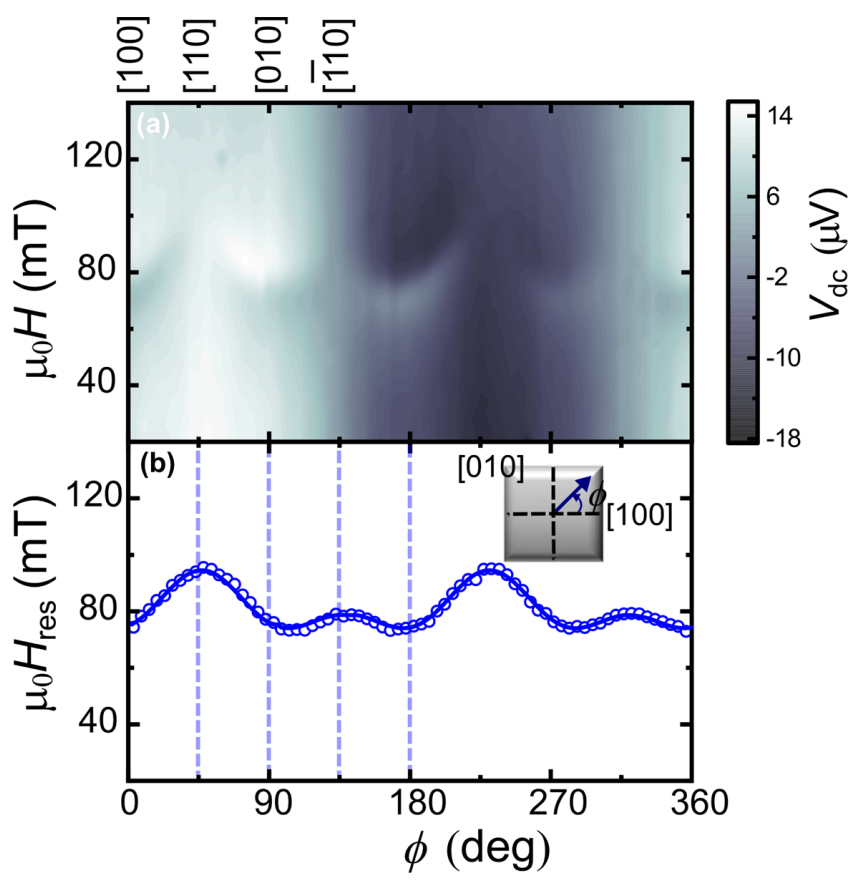

FIG. 3. (a) Two-dimensional color plot of FMR voltages $V_{\mathrm{dc}}$ measured from an in-plane rotational scan of external magnetic field as a function of applied magnetic field and angle. We use the microwave excitation frequency of $7 \mathrm{GHz}$. (b) FMR field $H_{\text {res }}$ as a function of the in-plane crystallographic angle. We plot results from fitting FMR scans.

$H_{2 \perp}, H_{2 \|}$, and $H_{4 \|}$ represent the out-of-plane uniaxial, in-plane uniaxial, and in-plane biaxial anisotropies, respectively, and $\phi$ is the angle between the magnetization vector $\mathbf{M}$ and the [100] crystallographic axis. In the experiments, we kept the frequency of the injected microwaves fixed while sweeping the external dc magnetic field. We also perform similar experiments by adding ac magnetic modulation, as described in the Supplemental Material [50], which provides results consistent with the dc magnetic field experiments. Figure 2(b) displays typical spin-orbit FMR data together with best-fit curves obtained using Eq. (2). The fit curves show a good quality of fitting, confirming the validity of the macrospin rectification model discussed above for our measurements.

\section{FMR ANALYSIS AND SPIN-ORBIT TORQUES}

By using the equations above, we are able to quantify the magnetic and spin-orbit parameters through a series of FMR measurements. Figure 3(a) shows $V_{\mathrm{dc}}$ measured for a bar oriented along the [110] direction as a function of $H$ and $\phi$. We can clearly identify a resonance in each scan, where $H_{\text {res }}$ depends on $\phi$ as shown in Fig. 3(b) and is used to deduce magnetic anisotropy parameters using our model. As summarized in Table I, we can identify that both uniaxial and biaxial (crystalline) anisotropies are present in the epitaxial NiMnSb films, consistent with VSM results [Fig. 1(c)]. These magnetic anisotropies are comparable with earlier reports [43,51]. Gerhard et al. quantified epitaxial NiMnSb films grown on an $\mathrm{InP} /(\mathrm{In}, \mathrm{Ga}) \mathrm{As}$ substrate with different
TABLE I. The magnetic parameters deduced from dc and ac FMR measurements.

\begin{tabular}{lcccccccc}
\hline \hline & \multicolumn{8}{c}{ Sample } \\
\cline { 2 - 10 } & \multicolumn{1}{c}{1} & \multicolumn{2}{c}{2} & \multicolumn{2}{c}{3} & \multicolumn{2}{c}{4} \\
\hline Direction & \multicolumn{2}{c}{$[1 \overline{1} 0]$} & {$[110]$} & \multicolumn{2}{c}{$[1 \overline{1} 0]$} & \multicolumn{2}{c}{$[110]$} \\
Modulation & $\mathrm{dc}$ & $\mathrm{ac}$ & $\mathrm{dc}$ & $\mathrm{ac}$ & $\mathrm{dc}$ & $\mathrm{ac}$ & $\mathrm{dc}$ & $\mathrm{ac}$ \\
$H_{2 \|}(\mathrm{mT})$ & -4 & -5 & -8 & -7 & -5 & -6 & -10 & -9 \\
$H_{4 \|}(\mathrm{mT})$ & 5 & 5 & 6 & 5 & 7 & 4 & 7 & 6 \\
$M_{\text {eff }}(\mathrm{mT})$ & 646 & 645 & 669 & 645 & 681 & 652 & 664 & 628 \\
\hline \hline
\end{tabular}

Mn concentrations and observed a large range of anisotropy changes in both terms [43], in particular showing that the uniaxial magnetic anisotropy becomes larger when the inplane lattice constant deviates from the stoichiometric value. Our samples have growth-induced strain that modifies the lattice constant away from the bulk value. Although it is difficult to make a direct quantitative comparison between $\mathrm{NiMnSb}$ on different substrates, we can note that the magnitude of $H_{2 \|}$ and $H_{4 \|}$ observed in our study matches fairly well with that measured by Gerhard et al. It is also possible that when epitaxial ferromagnetic films with growth-induced strain are lithographically patterned, uniaxial strain relaxation takes place, leading to a pattern-induced uniaxial magnetic anisotropy [52,53]. We would expect a similar anisotropy term in our NiMnSb film where the growth-induced compressive strain $\left(c / a=3.5 \times 10^{-4}\right)$ might relax when patterning a bar. However, when we compare bars patterned along the [110] and [1 10$]$ directions, we do not identify a clear presence of the pattern-induced term in the anisotropy within our detection limit since both devices exhibit very similar $H_{2 \|}$ and $H_{4 \|}$ values. It is worthwhile to note that a uniaxial anisotropy term is still present in epitaxial NiMnSb films even though the lattice constant of the two main axes (i.e., $a$ and $b$ ) match. This suggests the presence of additional symmetry lowering in the film plane from fourfold to twofold, which cannot be captured by our x-ray probe on crystallographic parameters. A similar result was previously observed in GaMnAs epitaxial thin films where it was attributed to a uniaxial anisotropy which can be understood by proposals of uniaxial distribution of $\mathrm{Mn}$ atoms that breaks the symmetry between the [110] and $[1 \overline{1} 0]$ orientations $[54,55]$. This can potentially lower an effective point group down to $2 \mathrm{~mm}$, allowing this uniaxial anisotropy to exist [55]. The Gilbert damping coefficient of our NiMnSb film is characterized as 0.007 using broadband FMR experiments.

The angular dependence of $V_{\text {sym }}$ and $V_{\text {asy }}$ reveals the spin-orbit torque parameters $[9,10]$. The fieldlike components of spin-orbit torques are associated with dissipative mechanisms $[5,6]$ and depend on the nonequilibrium distribution function of conduction electrons with specific spin textures in the Fermi surface. For NiMnSb, these components are parametrized by the in-plane components of the spin-orbit field, $h_{x}$ and $h_{y}$, which produce an asymmetric line shape of the resonance in $V_{\mathrm{dc}}$. By fitting the angular dependence of $V_{\text {asy }}$, we extract the magnitudes of $h_{x}$ and $h_{y}$ for each sample. Figures 4(a) and 4(b) include the angular dependence 
(a)

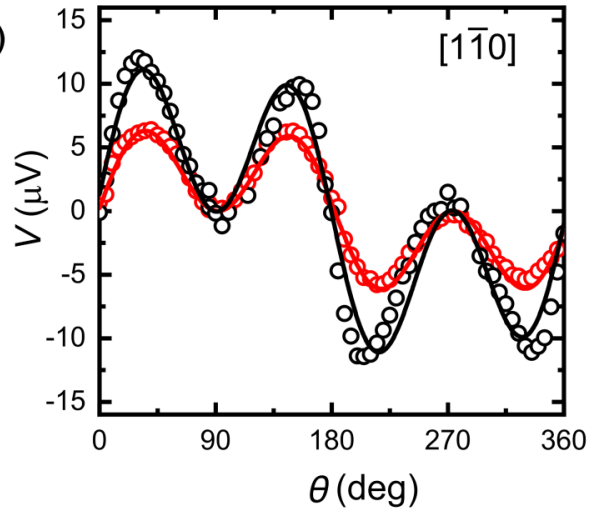

(c)
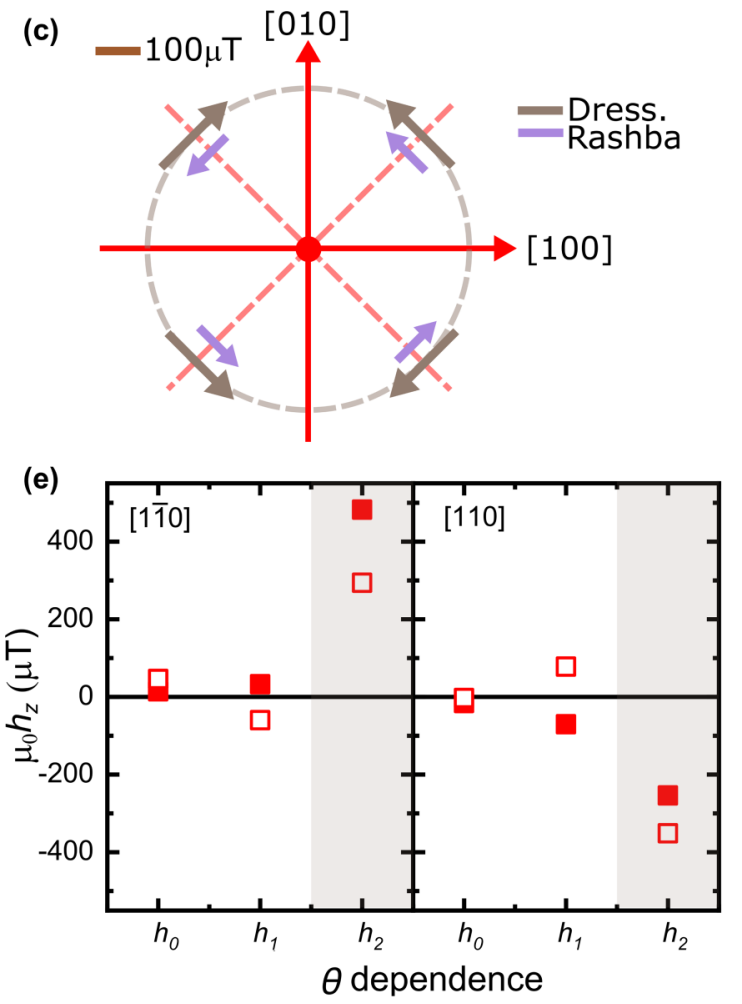

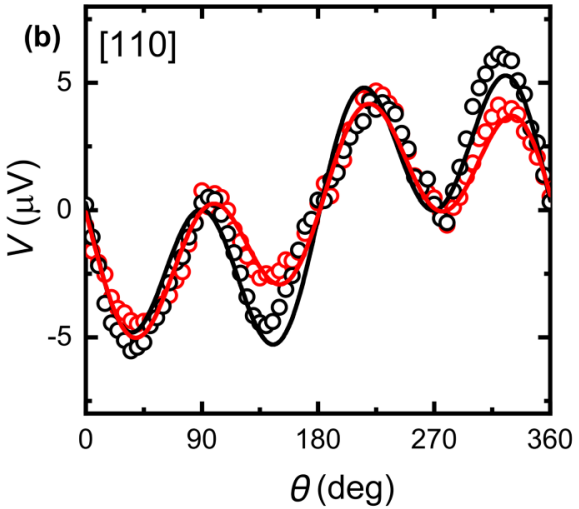

(d)
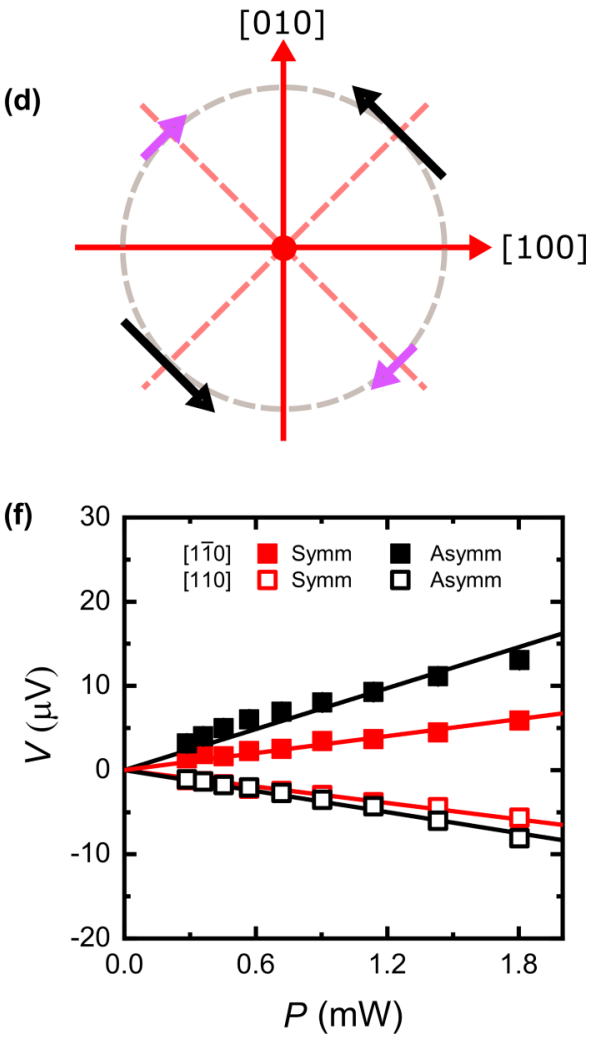

FIG. 4. (a) and (b) Angle dependence of the symmetric (open red circles) and antisymmetric (open black circles) components of the resonance measured for bars patterned along the (a) [1 $\overline{10}$ ] and (b) [110] crystal directions. The current density in the bar is $10^{10} \mathrm{~A} / \mathrm{m}^{2}$ and measured at a microwave excitation frequency of $7 \mathrm{GHz}$. (c) and (d) Plot of the magnitude and direction of the effective fields for (c) Dresselhaus and Rashba types of symmetry and (d) the sum of the two components. (e) $\theta$ dependence of the effective $h_{z}$ field. (f) Plot of magnitude of the voltage for the symmetric and antisymmetric components as a function of injected microwave powers.

of $V_{\text {asy }}$ (black dots) for [1 $\left.\overline{1} 0\right]$ and [110] samples, respectively, together with a best fit obtained by using Eq. (4). Both angular dependences show a clear $\sin 2 \theta \cos \theta$ symmetry, indicating that $h_{y}$ is the dominant component of the spin-orbit field. Furthermore, the sign flip between the [110] and [1피 samples, indicates that $h_{y}$ has the opposite signs for a current flowing along the [110] and [1ํㅣ directions. These observations are consistent with the previous work on MBE NiMnSb films [11]. By using the symmetry of the spin textures in momentum space for each spin-orbit term, we are able to decouple the Dresselhaus and Rashba components of the effective fields; the signs of the spin states for momentum along the [110] and [1 $1 \overline{1} 0]$ directions are opposite to each other in the Dresselhaus spin texture, while they are in the same direction in the Rashba spin texture, as depicted in Figs. 4(c). Hence, the effective fields from the Dresselhaus and Rashba terms $\left(h_{\mathrm{D}}\right.$ and $\left.h_{\mathrm{R}}\right)$ can be calculated as $h_{\mathrm{D}}=\left(h_{y, 110}-h_{y, 1 \overline{1} 0}\right) / 2$ and $h_{\mathrm{R}}=\left(h_{y, 110}+h_{y, 1 \overline{1} 0}\right) / 2$, respectively, where $h_{y, 110}\left(h_{y, 1 \overline{1} 0}\right)$ is the $h_{y}$ component extracted for the bar oriented along the

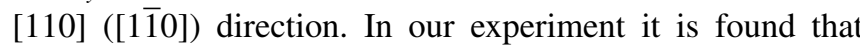
the Dresselhaus component is much greater than the Rashba one. When a zinc-blende crystal is distorted by elongating 
one of the [100], [010], and [001] orientations, the crystal point group is reduced from $\overline{4} 3 m$ to $\overline{4} 2 m$. In a system with $\overline{4} 2 m$ symmetry, the following spin-orbit term $H_{\mathrm{D}}$ is allowed, giving rise to the two-dimensional Dresselhaus spin texture, as shown in Fig. 4(c) (gray arrow):

$$
H_{\mathrm{D}}=\beta\left[J_{x} k_{x}\left(\epsilon_{y y}-\epsilon_{z z}\right)+\text { c.p. }\right] .
$$

Here, $\beta, J_{i}, k_{i}$, and $\epsilon_{i i}$ are the coefficient for this term, the $i$ component of the angular momentum of conduction carriers, the wave vector along the $i$ direction, and a diagonal element of the strain tensor in NiMnSb, respectively; c.p. represents cyclic permutation. When a zinc-blende crystal is under shear strain, meaning that an off-diagonal component of the strain tensor is nonzero, the point group is reduced to $m m 2$, which allows the following Rashba-type spin-orbit term, and the spin texture is illustrated in Fig. 4(c) (purple arrow):

$$
H_{\mathrm{R}}=\alpha_{\mathrm{R}}\left[\left(J_{x} k_{y}-J_{y} k_{x}\right) \epsilon_{x y}+\text { c.p. }\right] .
$$

Here, $\alpha_{\mathrm{R}}$ and $\epsilon_{i j}$ represent the coefficient for this Rashba term and an off-diagonal element of the strain tensor, respectively. Experimentally deduced values of $h_{\mathrm{D}}$ and $h_{\mathrm{R}}$ should be proportional to the strength of $H_{\mathrm{D}}$ and $H_{\mathrm{R}}$, respectively $[9,10]$. The growth-induced strain identified by our x-ray characterization produces finite values of $\left(\epsilon_{y y}-\epsilon_{z z}\right)$ as well as $\left(\epsilon_{z z}-\epsilon_{x x}\right)$, hence generating $h_{\mathrm{D}}$. However, we could not identify the presence of the off-diagonal strain, suggesting that this strain component is minute. Therefore, it is reasonable that the dominant effective field is $h_{\mathrm{D}}$ over $h_{\mathrm{R}}$.

We notice that in our samples there is a sizable component of $V_{\text {sym }}$. In our macrospin model, $V_{\text {sym }}$ leads to the presence of dampinglike torques which can also be interpreted as being induced by an out-of-plane component of the spin-orbit field $h_{z}$. This is because the magnetization precession induced by $h_{z}$ is phase shifted by $\pi / 2$ with respect to the one generated by the aforementioned fieldlike torques, leading to a line shape change into Lorentzian symmetric $V_{\text {sym }}$. In GaMnAs it was demonstrated that the current-induced dampinglike torques originate from the Berry curvature of the electronic band structures in GaMnAs [10]. Contrary to the previous report [11], in our case we observe non-negligible $V_{\text {sym }}$ components for both the [110] and [1̄0] samples, as shown in Figs. 4(a) and 4(b). In order to analyze the dampinglike torque component, we allow $h_{z}$ in Eq. (3) to be angle dependent as $h_{z}(\theta)=h_{0}+h_{1} \sin \theta+h_{2} \cos \theta$. By comparing the general forms of the fieldlike and dampinglike torques, i.e., $\mathbf{m} \times(m \times s)$ and $\mathbf{m} \times h$, we can interpret that the $z$ component of $\mathbf{h}$ can be written as $h_{z}=m_{y} s_{x}-m_{x} s_{y}$. Using the definition of $\theta$ in our analysis, we find that $\left(m_{x}, m_{y}\right)=M_{\mathrm{s}}(\cos \theta, \sin \theta)$ are the in-plane components of the magnetization, while $\left(s_{x}\right.$, $s_{y}$ ) are the components of the current-induced spin polarization. Substituting this into the previous equation, we find $h_{z}=$ $M_{\mathrm{s}}\left(s_{x} \sin \theta-s_{y} \cos \theta\right)$. Therefore, for a $s_{x}\left(s_{y}\right)$ spin polarization, $h_{z}(\theta)$ has sine (cosine) symmetry. Clearly, our observation of $V_{\text {sym }}$ in Figs. 4(a) and 4(b) displays $\sin 2 \theta \cos \theta$ symmetry, suggesting that $h_{z}(\theta)$ must have $\cos \theta$ symmetry upon the in-plane rotation of $M$. This, together with the aforementioned analysis, leads to the conclusion that the predominant contribution of the dampinglike torques is from $s_{y}$ spin polarization in momentum space. In Fig. 4(e) we plot $h_{0}, h_{1}$, and $h_{2}$. A sign
TABLE II. SOT effective fields for various material systems. All of them have been scaled by a current density of $j=$ $1 \times 10^{10} \mathrm{~A} / \mathrm{m}^{2}$. The following unit is used for effective fields: $h_{\mathrm{FL}, \mathrm{DL}} / j\left[\mathrm{mT} /\left(10^{10} \mathrm{~A} / \mathrm{m}^{2}\right)\right]$. Note that a more completed list is avail-

\begin{tabular}{|c|c|c|c|}
\hline Structure type & Name & $h_{F L \|} / \mathrm{j}$ & $h_{D L \|} /$ \\
\hline \multirow[t]{3}{*}{ Bulk ferromagnets } & $(\mathrm{Ga}, \mathrm{Mn})$ As [10] & -2.01 & -1.2 \\
\hline & $\mathrm{NiMnSb[11]}$ & -0.06 & \\
\hline & $\mathrm{NiMnSb}^{\mathrm{a}}$ & -0.31 & -0 \\
\hline \multirow[t]{4}{*}{ Nonmagnetic metals } & $\mathrm{Pt} / \mathrm{Co} / \mathrm{AlO}_{x}[26]$ & 0.4 & -0.6 \\
\hline & $\mathrm{Ti} / \mathrm{CoFe} / \mathrm{Pt}[30]$ & -0.03 & 0.3 \\
\hline & $\mathrm{Ta} / \mathrm{CoFeB} / \mathrm{MgO}[31]$ & -0.21 & 0 . \\
\hline & $\mathrm{Pd} / \mathrm{Co} / \mathrm{AlO}_{x}[32]$ & 0.07 & 0. \\
\hline Antiferromagnets & $\mathrm{IrMn}_{3} / \mathrm{CoFeB} / \mathrm{MgO}$ [33] & 0.07 & -0 \\
\hline Semiconductors & (Ga,Mn)As/Fe [34] & 0.03 & -0 \\
\hline Topological insulators & $\mathrm{Mn}_{0.4} \mathrm{Ga}_{0.6} / \mathrm{Bi}_{0.9} \mathrm{Sb}_{0.1}$ [57] & & -23 \\
\hline
\end{tabular}
able in Ref. [56].

${ }^{\mathrm{a}} \mathrm{NiMnSb}$ measured in this paper.

change in $h_{2}$ between the [110] and [110] samples is consistent with a sign flip of $s_{y}$ and therefore with the Dresselhaus symmetry of the spin texture in $\mathrm{NiMnSb}$, as also found in our previous analysis of the fieldlike torques. Finally, we show the linear dependence of the rectified longitudinal voltage $V_{\mathrm{dc}}$ on microwave power in Fig. 4(f). This dependence is consistent with our spin-texture model since $V_{\text {sym }} \propto I^{2} \propto P$, where $h_{i}$ ( $i=x, y$, and $z) \propto I$ in Eqs. (3) and (4).

To complete our analysis, it is useful to compare the values of the spin-orbit-torque (SOT) in our sputtered $\mathrm{NiMnSb}$ films to those reported in previous works. We use an effective current-induced field size $\left(h_{\mathrm{FL}}\right.$ and $h_{\mathrm{DL}}$ for fieldlike and dampinglike fields, respectively) per a normalized current density of $j=1 \times 10^{10} \mathrm{~A} / \mathrm{m}^{2}$ as a figure of merit for this analysis. Our NiMnSb films show $h_{\mathrm{FL}}=0.31 \mathrm{mT}$ and $h_{\mathrm{DL}}=$ $0.48 \mathrm{mT}$. These are larger than $h_{\mathrm{FL}}$ in MBE-grown NiMnSb $h_{\mathrm{FL}}=0.06 \mathrm{mT}$ [11]. This result has a technological interest since sputtering techniques are compatible with industrial processes and less costly. Furthermore, we also highlight that our $h_{\mathrm{FL} / \mathrm{DL}}$ values are comparable to those reported in heavymetal/ferromagnet bilayer systems as listed in Table II. For completeness, we also list the spin-orbit field size measured in sputtering-grown topological insulators, which is as large as $230 \mathrm{mT}$ at $j=1 \times 10^{10} \mathrm{~A} / \mathrm{m}^{2}$ as reported by Khang et al. [57] very recently.

\section{CONCLUSIONS}

In this study, we presented measurements of spin-orbit torques in sputter-deposited epitaxial NiMnSb on a GaAs substrate. X-ray characterization revealed that $\mathrm{NiMnSb}$ grows epitaxially with a cube-on-cube orientation relationship of $\mathrm{GaAs}(001) / / \mathrm{NiMnSb}(001)$ and GaAs[100]//NiMnSb[100]. A growth-induced strain is present, which distorts the lattice constants as $a=b=0.5937 \pm 0.0002 \mathrm{~nm}$ and $c=0.5921 \pm$ $0.0002 \mathrm{~nm}$, giving $c / a=0.997 \pm 0.0002$. We characterized the spin-orbit torques by two current-induced FMR methods and found that the torques originate from the spin-orbit interaction within the tetragonally distorted NiMnSb crystal. Furthermore, both fieldlike and dampinglike torques were 
found to be present in our samples, which is different from spin-orbit torques in MBE-grown NiMnSb. We found that the effective field generation efficiency of our sputteringgrown NiMnSb is comparable to those reported from heavymetal/ferromagnet bilayer systems.

\section{ACKNOWLEDGMENTS}

We are grateful to S. Mitani for his technical supports for sputtered film preparation, as well as $\mathrm{K}$. Hanzawa and $\mathrm{H}$. Hiramatsu for their efforts to grow NiMnSb films.
[1] V. M. Edelstein, Solid State Commun. 73, 233 (1990).

[2] L. S. Levitov, Yu. V. Nazarov, and G. M. Eliashberg, Zh. Eksp. Teor. Fiz. 88, 229 (1985) [Sov. Phys. JETP 61, 133 (1985)].

[3] E. L. Ivchenko and S. Ganichev, in Spin Physics in Semiconductors, edited by M. I. Dyakonov (Springer, New York, 2008), p. 263.

[4] A. Y. Silov, P. A. Blajnov, J. H. Wolter, R. Hey, K. H. Ploog, and N. S. Averkiev, Appl. Phys. Lett. 85, 5929 (2004).

[5] B. A. Bernevig and O. Vafek, Phys. Rev. B 72, 033203 (2005).

[6] A. Manchon and S. Zhang, Phys. Rev. B 78, 212405 (2008).

[7] A. Chernyshov, M. Overby, X. Liu, J. K. Furdyna, Y. LyandaGeller, and L. P. Rokhinson, Nat. Phys. 5, 656 (2009).

[8] M. Endo, F. Matsukura, and H. Ohno, Appl. Phys. Lett. 97, 222501 (2010).

[9] D. Fang, H. Kurebayashi, J. Wunderlich, K. Vyborny, L. P. Zarbo, R. P. Campion, A. Casiraghi, B. L. Gallagher, T. Jungwirth, and A. J. Ferguson, Nat. Nanotechnol. 6, 413 (2011).

[10] H. Kurebayashi, J. Sinova, D. Fang, A. C. Irvine, T. D. Skinner, J. Wunderlich, V. Novák, R. P. Campion, B. L. Gallagher, E. K. Vehstedt, L. P. Zârbo, K. Výborný, A. J. Ferguson, and T. Jungwirth, Nat. Nanotechnol. 9, 211 (2014).

[11] C. Ciccarelli, L. Anderson, V. Tshitoyan, A. J. Ferguson, F. Gerhard, C. Gould, L. W. Molenkamp, J. Gayles, J. Železný, L. Šmejkal, Z. Yuan, J. Sinova, F. Freimuth, and T. Jungwirth, Nat. Phys. 12, 855 (2016).

[12] P. Wadley et al., Science 351, 587 (2016).

[13] L. Chen, M. Decker, M. Kronseder, R. Islinger, M. Gmitra, D. Schuh, D. Bougeard, J. Fabian, D. Weiss, and C. H. Back, Nat. Commun. 7, 13802 (2016).

[14] D. MacNeill, G. M. Stiehl, M. H. D. Guimaraes, R. A. Buhrman, J. Park, and D. C. Ralph, Nat. Phys. 13, 300 (2017).

[15] M. I. Dyakonov and V. I. Perel, Phys. Lett. A 35, 459 (1971).

[16] J. E. Hirsch, Phys. Rev. Lett. 83, 1834 (1999).

[17] S. Murakami, N. Nagaosa, and S. C. Zhang, Science 301, 1348 (2003).

[18] Y. Kato, R. C. Myers, A. C. Gossard, and D. D. Awschalom, Science 306, 1910 (2004).

[19] J. Wunderlich, B. Kaestner, J. Sinova, and T. Jungwirth, Phys. Rev. Lett. 94, 047204 (2005).

[20] J. Sinova, S. O. Valenzuela, J. Wunderlich, C. H. Back, and T. Jungwirth, Rev. Mod. Phys. 87, 1213 (2015).

[21] K. Ando, S. Takahashi, K. Harii, K. Sasage, J. Ieda, S. Maekawa, and E. Saitoh, Phys. Rev. Lett. 101, 036601 (2008).

[22] L. Q. Liu, T. Moriyama, D. C. Ralph, and R. A. Buhrman, Phys. Rev. Lett. 106, 036601 (2011).

[23] I. M. Miron, G. Gaudin, S. Auffret, B. Rodmacq, A. Schuhl, S. Pizzini, J. Vogel, and P. Gambardella, Nat. Mater. 9, 230 (2010).

[24] I. M. Miron, K. Garello, G. Gaudin, P. J. Zermatten, M. V. Costache, S. Auffret, S. Bandiera, B. Rodmacq, A. Schuhl, and P. Gambardella, Nature (London) 476, 189 (2011).
[25] J. Kim, J. Sinha, M. Hayashi, M. Yamanouchi, S. Fukami, T. Suzuki, S. Mitani, and H. Ohno, Nat. Mater. 12, 240 (2012).

[26] K. Garello, I. M. Miron, C. O. Avci, F. Freimuth, Y. Mokrousov, S. Blügel, S. Auffret, O. Boulle, G. Gaudin, and P. Gambardella, Nat. Nanotechnol. 8, 587 (2013).

[27] S. Emori, T. Nan, A. M. Belkessam, X. Wang, A. D. Matyushov, C. J. Babroski, Y. Gao, H. Lin, and N. X. Sun, Phys. Rev. B 93, 180402(R) (2016).

[28] S. Fukami, T. Anekawa, C. Zhang, and H. Ohno, Nat Nanotechnol. 11, 621 (2016).

[29] Y. W. Oh, S. H. C. Baek, Y. M. Kim, H. Y. Lee, K. D. Lee, C. G. Yang, E. S. Park, K. S. Lee, K. W. Kim, G. Go, J. R. Jeong, B. C. Min, H. W. Lee, K. J. Lee, and B. G. Park, Nat. Nanotechnol. 11, 878 (2016).

[30] X. Fan, H. Celik, J. Wu, C. Ni, K. J. Lee, V. O. Lorenz, and J. Q. Xiao, Nat. Commun. 5, 3042 (2014).

[31] C. O. Avci, K. Garello, C. Nistor, S. Godey, B. Ballesteros, A. Mugarza, A. Barla, M. Valvidares, E. Pellegrin, A. Ghosh, I. M. Miron, O. Boulle, S. Auffret, G. Gaudin, and P. Gambardella, Phys. Rev. B 89, 214419 (2014).

[32] A. Ghosh, K. Garello, C. O. Avci, M. Gabureac, and P. Gambardella, Phys. Rev. Appl. 7, 014004 (2017).

[33] D. Wu, G. Yu, C. T. Chen, S. A. Razavi, Q. Shao, X. Li, B. Zhao, K. L. Wong, C. He, Z. Zhang, P. Khalili Amiri, and K. L. Wang, Appl. Phys. Lett. 109, 222401 (2016).

[34] T. D. Skinner, K. Olejník, L. K. Cunningham, H. Kurebayashi, R. P. Campion, B. L. Gallagher, T. Jungwirth, and A. J. Ferguson, Nat. Commun. 6, 6730 (2015).

[35] K. Cai, M. Yang, H. Ju, S. Wang, Y. Ji, B. Li, K. W. Edmonds, Y. Sheng, B. Zhang, N. Zhang, S. Liu, H. Zheng, and K. Wang, Nat. Mater. 16, 712 (2017).

[36] A. Pospischil, M. M. Furchi, and T. Mueller, Nat. Nanotechnol. 9, 257 (2014).

[37] Y. Cao, Y. Sheng, K. W. Edmonds, Y. Ji, H. Zheng, and K. Wang, Adv. Mater. 32, 1907929 (2020).

[38] K. M. D. Hals and A. Brataas, Phys. Rev. B 88, 085423 (2013).

[39] J. Zelezny, H. Gao, A. Manchon, F. Freimuth, Y. Mokrousov, J. Zemen, J. Mašek, J. Sinova, and T. Jungwirth, Phys. Rev. B 95, 014403 (2017).

[40] C. Felser, L. Wollmann, S. Chadov, G. H. Fecher, and S. S. P. Parkin, APL Mater. 3, 041518 (2015).

[41] M. J. Otto, R. A. M. Van Woerden, P. J. Van Der Valk, J. Wijngaard, C. F. Van Bruggen, and C. Haas, J. Phys.: Condens. Matter 1, 2341 (1989).

[42] P. Dürrenfeld, F. Gerhard, J. Chico, R. K. Dumas, M. Ranjbar, A. Bergman, L. Bergqvist, A. Delin, C. Gould, L. W. Molenkamp, and J. Åkerman, Phys. Rev. B 92, 214424 (2015).

[43] F. Gerhard, C. Schumacher, C. Gould, and L. W. Molenkamp, J. Appl. Phys. 115, 094505 (2014).

[44] Half-Metallic Materials and Their Properties, edited by C. Y. Fong, J. E. Pask, L. H. Yang (Imperial College Press, London, 2013). 
[45] M. E. Straumanis and C. D. Kim, Acta Crystallogr. 19, 256 (1965).

[46] L. Ritchie, G. Xiao, Y. Ji, T. Y. Chen, C. L. Chien, M. Zhang, J. Chen, Z. Liu, G. Wu, and X. X. Zhang, Phys. Rev. B 68, 104430 (2003).

[47] D. Orgassa and H. Fujiwara, J. Appl. Phys. 87, 5870 (2000).

[48] J. J. Attema, C. M. Fang, L. Chioncel, G. A. de Wijsand, A. I. Lichtenstein, and R. A. de Groot, J. Phys.: Condens. Matter 16, S5517 (2004).

[49] B. Kwon, Y. Sakuraba, H. Sukegawa, S. Li, G. Qu, T. Furubayashi, and K. Hono, J. Appl. Phys. 119, 023902 (2016).

[50] See Supplemental Material at http://link.aps.org/supplemental/ 10.1103/PhysRevMaterials.5.014413 for supporting experimental details and similar measurements by ac magnetic modulation.

[51] A. Koveshnikov, G. Woltersdorf, J. Q. Liu, B. Kardasz, O. Mosendz, B. Heinrich, K. L. Kavanagh, P. Bach, A. S. Bader,
C. Schumacher, C. Rüster, C. Gould, G. Schmidt, L. W. Molenkamp, and C. Kumpf, J. Appl. Phys. 97, 073906 (2005).

[52] J. Wenisch, C. Gould, L. Ebel, J. Storz, K. Pappert, M. J. Schmidt, C. Kumpf, G. Schmidt, K. Brunner, and L. W. Molenkamp, Phys. Rev. Lett. 99, 077201 (2007).

[53] C. S. King, J. Zemen, K. Olejník, L. Horák, J. A. Haigh, V. Novák, A. Irvine, J. Kučer, V. Holý, R. P. Campion, B. L. Gallagher, and T. Jungwirth, Phys. Rev. B 83, 115312 (2011).

[54] M. Kopecký, J. Kub, F. MácA, J. Mažek, O. Pacherová, A. W. Rushforth, B. L. Gallagher, R. P. Campion, V. Novák, and T. Jungwirth, Phys. Rev. B 83, 235324 (2011).

[55] M. Birowska, C. Liwa, J. A. Majewski, and T. Dietl, Phys. Rev. Lett. 108, 237203 (2012).

[56] A. Manchon, J. Železný, I. M. Miron, T. Jungwirth, J. Sinova, A. Thiaville, K. Garello, and P. Gambardella, Rev. Mod. Phys. 91, 035004 (2019).

[57] N. H. D. Khang, Y. Ueda, and P. N. Hai, Nat. Mater. 17, 808 (2018). 